\title{
EFFECT OF Staphylococcus epidermidis ON Pseudomonas aeruginosa BIOFILM IN MIXED-SPECIES CULTURE
}

\author{
Suhaga Dohare ${ }^{1}$, Devendra Singh ${ }^{1}$, Deepmala Sharma ${ }^{2}$, Vishnu Agarwal $^{1 *}$ \\ ${ }^{1}$ Department of Biotechnology Motilal Nehru National Institute of Technology Allahabad-211004, India \\ ${ }^{2}$ Department of Mathematics, National Institute of Technology, Raipur-492010, India
}

Received - March 16, 2021; Revision - May 21, 2021; Accepted - June 11, 2021

Available Online - June 25, 2021

DOI: http://dx.doi.org/10.18006/2021.9(3).325.334

\author{
KEYWORDS \\ Staphylococcus epidermidis \\ Pseudomonas aeruginosa \\ Mixed culture \\ Planktonic \\ Biofilm \\ Co-survivability
}

\begin{abstract}
Staphylococcus epidermidis and Pseudomonas aeruginosa, are clinically relevant pathogens that often produce biofilms. To investigate the co-survivability of $S$. epidermidis and $P$. aeruginosa in mixed cultures biofilm and planktonic form, it is important to understand more about the interspecies interaction of both species. The interspecies interaction was analyzed using streak and drop agar plate assay, cell viability assay (CFU), spectrophotometry-based method, and microscopic analysis. The findings suggest that both cells and supernatant of $P$. aeruginosa inhibit the planktonic growth of $S$. epidermidis. The cell viability result shows that PAO1 biofilm cells were decreased by $88 \%$, and SE biofilm cells were increased by $75 \%$ concerning their control. Opposite to the P. aeruginosa, the $S$. epidermidis biofilm and EPS matrix were found to increase in mixed culture biofilm, which was further confirmed by microscopic analysis. In contrast, differential agar media result shows that the reduction in the biofilm $(\mathrm{CFU} / \mathrm{ml})$ of $P$. aeruginosa is independent of $S$. epidermidis cells concentration. Finally, the effect of the supernatant on biofilm was investigated, and it found that S. epidermidis biofilm was enhanced while $P$. aeruginosa biofilm was reduced in the presence of partner bacterial supernatant, which indicated that $S$. epidermidis in biofilm mode could hinder the biofilm formation of $P$. aeruginosa. The outcomes show that the culture supernatant of $S$. epidermidis can be used to prevent $P$. aeruginosa associated biofilm infections.
\end{abstract}

* Corresponding author

E-mail: vishnua@mnnit.ac.in (Dr. Vishnu Agarwal)

Peer review under responsibility of Journal of Experimental Biology and Agricultural Sciences.

Production and Hosting by Horizon Publisher India [HPI] (http://www.horizonpublisherindia.in/).

All rights reserved.
All the articles published by Journal of Experimental Biology and Agricultural Sciences are licensed under a Creative Commons Attribution-NonCommercial 4.0 International License Based on a work at www.jebas.org.

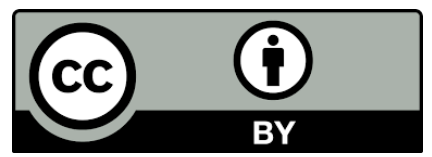




\section{Introduction}

Biofilm is the consortium of multiple microbial species enclosed with a tightly packed exopolysaccharide matrix known as mixed culture biofilm. This mixed microbial consortium competes for common nutritional resources for survival in the hostile environment (Rogers et al., 2010). It is expected that significant interaction takes place between coexisting microbial species. This coexisting interaction may be mutualistic or antagonistic (Cai et al., 2019). Earlier it was reported that different bacteria coexist in cystic fibrosis (CF), implant-related infection, and contact lenses that contribute to the pathogenesis of the disease (Morales et al., 2013; Baldan et al., 2014). As in the biofilm mode of growth, microorganisms of different species exist together and interact with each other agonistically. Therefore analysis of single-species biofilms will not be sufficient for the treatment of mixed culture biofilm-related infections (Hibbing et al., 2010; Baldan et al., 2014) as virulence factors produced by $S$. epidermidis (SE) is comparatively less than $S$. aureus and are human-friendly bacterium found in the skin microflora and sebaceous gland. It is reported as a harmless commensal bacterium, but it becomes virulent when it reaches inside the human body through the use of indwelling medical devices (Foster, 2020; Joshi et al., 2021). It can be responsible for causing nosocomial infection, blood infection, respiratory tract infection, urinary tract infection, and endocarditis. S. epidermidis is found resistant against several antibiotics like trimethoprim, penicillin, ciprofloxacin, clindamycin, levofloxacin, erythromycin, gentamicin, and mupirocin, which nowadays becomes a serious issue in hospitals (Chabi \& Momtaz, 2019). It is believed that being present in the skin protects against the other pathogenic bacteria by competing with them (Otto, 2013). $P$. aeruginosa (PAO1) is the most prevalent and more virulent Gramnegative bacterium found in polymicrobial infections, including cystic fibrosis (CF) and diabetic foot and surgical infections. In 2017, P. aeruginosa evolved as one of the life-threatening pathogen (Thi et al., 2020). According to a WHO report, $P$. aeruginosa was listed as a pathogen of main concern for the development of a new treatment strategy (WHO 2017). $P$. aeruginosa possesses the intrinsic resistant ability to the existing antibiotics (Pang et al., 2019). The present study was carried out to understand how these bacterial species influence each other in mixed planktonic culture and biofilm communities.

\section{Materials and Methods}

\subsection{Bacteria and growth conditions}

This examination utilized the bacterial strain $P$. aeruginosa PAO1 and S. epidermidis 435 that were acquired from the MTCC, IMTECH, Chandigarh, India. Propidium Iodide (PI) and FITC-con A were purchased from Sigma Aldrich, India. P. aeruginosa was cultivated at $\mathrm{pH} 7$ in Luria-Bertani (LB) medium, S. epidermidis strain was cultivated in Tryptic Soya Broth (TSB), and both were maintained at $37^{\circ} \mathrm{C}$ for $24 \mathrm{~h}$. Mannitol salt agar (S. epidermidis) and pseudomonas isolation agar ( $P$. aeruginosa) was used as selective media for the viable count.

\subsection{Planktonic interaction studies}

\subsubsection{Interaction on an agar plate and liquid medium}

Each strain's inhibitory effect was evaluated after $24 \mathrm{~h}$ growth by streak agar assay and drop agar assay. Briefly, $1 \%$ of $\mathrm{OD}_{600} 0.5$ cultures of both species PAO1 and SE was inoculated into fresh, sterilized LB broth media and kept in a shaking incubator for $24 \mathrm{~h}$ at $37^{\circ} \mathrm{C}$. In streak agar plate assay, an equal amount of each culture $(20 \mu 1$ fresh culture) was spread on half of the agar plates. In drop agar assay, $100 \mu \mathrm{l}$ of SE's $24 \mathrm{~h}$ grown culture as uniformly spread on the LB agar plate using a cotton swab. Then drops containing $10 \mu \mathrm{l}$ of PAO1 culture were placed in the center of the agar surface, and all agar plates were incubated for $24 \mathrm{~h}$ at $37^{\circ} \mathrm{C}$. The same step was repeated for spotting SE culture on the PAO1 lawn. To estimate the growth of PAO1 in the presence of SE cells, free supernatant, and vice-versa. PAO1 was grown in the presence of SE culture supernatant $(0.5,1.5,2.5,5$, and $15 \%)$ in polystyrene 96-well microtiter plates (Tarson) at $37^{\circ} \mathrm{C}$ in a shaking incubator and at different time interval $(0,1,2,4,6,8$ and $24 \mathrm{~h})$ absorbance was measured $\left(\mathrm{A}_{600} \mathrm{~nm}\right)$ (Hoffman et al., 2006; Fugère et al., 2014).

\subsubsection{Preparation of mixed culture consortia}

Overnight grown cultures of SE and PAO1 were centrifuged at $4^{0} \mathrm{C}$ and 1000rpm for $15 \mathrm{~min}$, the pellet was rinsed with PBS (Phosphate buffer saline, $\mathrm{pH}$ 7.4), and desired $\mathrm{OD}_{600}$ was set as per experimental requirements. To access the interspecies interaction in mixed culture biofilm, varying cell concentration of SE 0.5, 0.6, $0.7,0.8$, and 0.9 OD that was corresponding to $5.5 \times 10^{9}, 6 \times 10^{9}$, $7 \times 10^{9}, 8.5 \times 10^{9}$, and $1.5 \times 10^{10} \mathrm{CFU} / \mathrm{ml}$ respectively were diluted in TSB (1:100) and mixed with 1:100 diluted $0.5 \mathrm{OD}_{600}$ culture of PAO1 $\left(5.5 \times 10^{9} \mathrm{CFU} / \mathrm{ml}\right)$, and different mixed cultures were termed as M1, M2, M3, M4, M5 respectively (Iwase et al., 2010).

\subsection{Mix culture biofilm studies}

\subsubsection{Co-survival study in a biofilm system}

Briefly, for mixed culture biofilm,100 $\mu 1$ standardized inoculums of PAO1 were inoculated in a 96-well polystyrene plate with $100 \mu 1$ of different cells concentration of SE. For control, 100 $\mu 1$ of standardized inoculums of single species were inoculated in a polystyrene plate (96-well microtiter plate) with $100 \mu \mathrm{l}$ of sterile TSB medium. Then these plates were incubated for $24 \mathrm{~h}$ at $37^{\circ} \mathrm{C}$ in a static condition. The wells were washed with sterile PBS (three times), air-dried, and stained with $0.1 \%$ crystal violet $(\mathrm{CV})$ for 15 
$\min$. Then, the plate was rinsed with distilled water and air-dried And finally, in glacial acetic acid (30\%), the stained biofilm was resuspended, and absorbance was recorded at 570nm (Fugère et al., 2014).

\subsubsection{Microscopic Analysis}

For the microscopic analysis petri-dishes (12mm, Borosil) was used and biofilm formed on the glass coverslip containing $2.5 \mathrm{ml}$ of diluted inoculums of single culture with $2.5 \mathrm{ml}$ of sterilized TSB for control biofilm and $2.5 \mathrm{ml}$ diluted inoculums of PAO1 mixed with $2.5 \mathrm{ml}$ of each cells concentration of SE for mixed cultures biofilm and incubated in static condition for $24 \mathrm{~h}$. This was followed by discarding the free planktonic cells, washed with PBS (three times) and stained with $0.1 \% \mathrm{CV}$ dye, removed extra stained again through washing without disturbing biofilm, then the biofilms coverslip were placed on a glass slide, dried, and observed under the light microscope and images were taken at 100X (Nithya et al., 2010).

\subsubsection{Scanning Electron Microscopy (SEM)}

A scanning electron microscopic study was carried to examine the cells of both bacteria in the mixed biofilm. Briefly, the mixed biofilm formed (M1-M5) on the coverslip as described above, and biofilms were fixed with paraformaldehyde (2\%)-glutaraldehyde $(2.5 \%)$ solution formed in $0.2 \mathrm{M}$ phosphate buffer $(\mathrm{pH}$ 7.4). Fixed biofilms on coverslips were dehydrated with ethanol series of 10 , $30,50,70$, and $100 \%$ for $10 \mathrm{~min}$, and after that, each sample was put at room temperature overnight to dry completely. The resultant samples were sputtered with gold-palladium (20:80) and visualized under a Scanning electron microscope (Sigma) (Fischer et al. 2012).

\subsubsection{Viable count assay}

The biofilm formed on the coverslip surface was detached by the sonication procedure, as previously described by Kobayashi et al. (2009). The biofilm was sonicated in $1 \mathrm{ml}$ of PBS (pH 7.4) for 5 minutes, followed by vortexing for 2 minutes. After sonication and vortexing, the suspension is subsequently diluted $10^{5}$ times and inoculated to differential selective media (Mannitol salt agar for $S$. epidermidis and pseudomonas isolation agar for $P$. aeruginosa) and incubated for $24 \mathrm{~h}$ at $37^{\circ} \mathrm{C}$.

\subsubsection{Effect of bacterial supernatant on interspecies biofilm}

To analyze the effect of SE culture supernatant on PAO1 biofilm formation, PAO1culture was inoculated with various SE culture concentrations supernatant to the wells of polystyrene microtiter plates $(0.5,1.5,2.5,5$, and $15 \%)$ and incubated for $24 \mathrm{~h}$ at $37^{\circ} \mathrm{C}$. After incubation, the biofilm was stained with $\mathrm{CV}$ dye, as described above. The same procedure was followed to investigate the impact of the PAO1 supernatant on SE biofilm.

\subsubsection{Fluorescent Microscopic analysis}

For fluorescent microscopy analysis, PAO1 was developed on a glass coverslip in the absence or presence of cell-free supernatant SE435 (0 to 15\%) in a static condition and vice versa. After $24 \mathrm{~h}$ of incubation, the biofilm formed was gently washed with PBS, fixed with paraformaldehyde, and total biofilm cells were stained with PI $(10 \mu \mathrm{g} / 100 \mu \mathrm{l})$ (Kalia et al., 2020), and EPS was stained with FITCConA (Singh et al., 2017)(50 $\mu \mathrm{g} / \mathrm{ml}$ in PBS, pH 7.4) containing $\mathrm{Ca}^{2+}(0.1 \mathrm{mM})$. By using an OLYMPUS BX51 fluorescent microscope, fluorescent microscopy was performed, and the images were taken at $20 \mathrm{X}$.

\subsection{Statistical Analysis}

One-way ANOVA, "Tukey's Multiple Comparison Test," was used for statistical analyses. Error bars indicate the standard deviations for three measurements. A value at $\mathrm{p}<0.05$ was considered statistically significant.

\section{Results}

\section{1 $P$. aeruginosa inhibits $S$. epidermidis growth in planktonic} form

The interaction between $P$. aeruginosa (PAO1) and $S$. epidermidis (SE) was analyzed on the agar plate (Figure 1). When equal inoculums of an overnight culture of both species were grown in equal half of the agar plate, it was reported that PAO1 inhibited the growth of SE. The clear inhibitory boundary was observed, as shown in Figure 1a. Zone of inhibition was found around the spot inoculated PAO1, and instead of lawn, spotted colonies of SE were observed, as shown in Figure 1b. On the other hand, SE did not show the zone of inhibition around the point inoculums on a lawn of PAO1 (Figure 1c). Furthermore, to test whether this inhibition was limited to the cell to cell contact, filter-sterilized supernatant of PAO1 were incubated with SE cells and vice versa (Figure 2). It was found that the growth of SE was inhibited by supernatant $(0.5-15 \%)$ of PAO1 in a concentration-dependent manner, as shown in Figure 2a. However, the supernatant of SE did not affect PAO1 growth (Figure 2b). The maximum inhibition was observed at $15 \%$ supernatant concentration in $24 \mathrm{~h}$. Net $0.7,0.8,1,1.1$, and 1.3 fold reduction in the growth of SE was observed at 0.5, 1.5, $2.5,5$, and $15 \%$ supernatant of PAO1, respectively. 

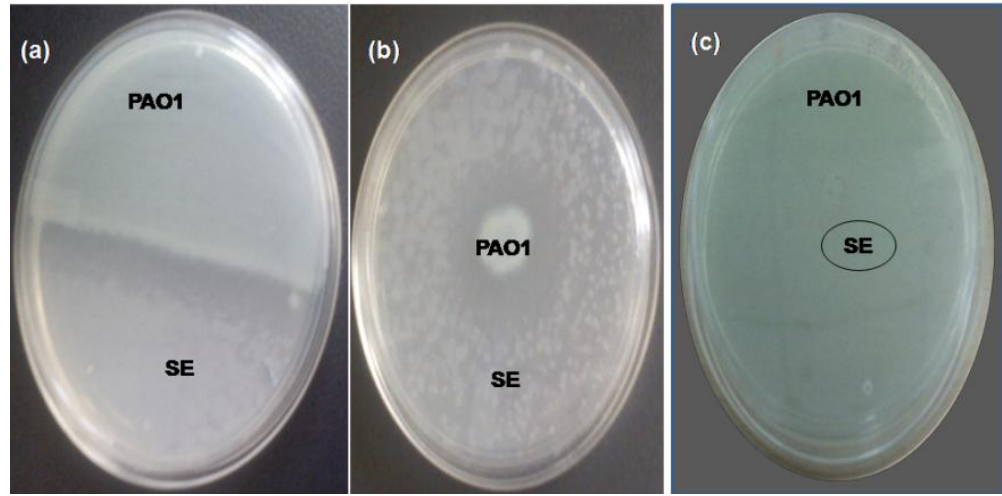

Figure $1 S$. epidermidis growth inhibition by $P$. aeruginosa on the agar plate. (a) streak agar plate (b) $P$. aeruginosa drop agar plate (c) S. epidermidis drop agar plate.
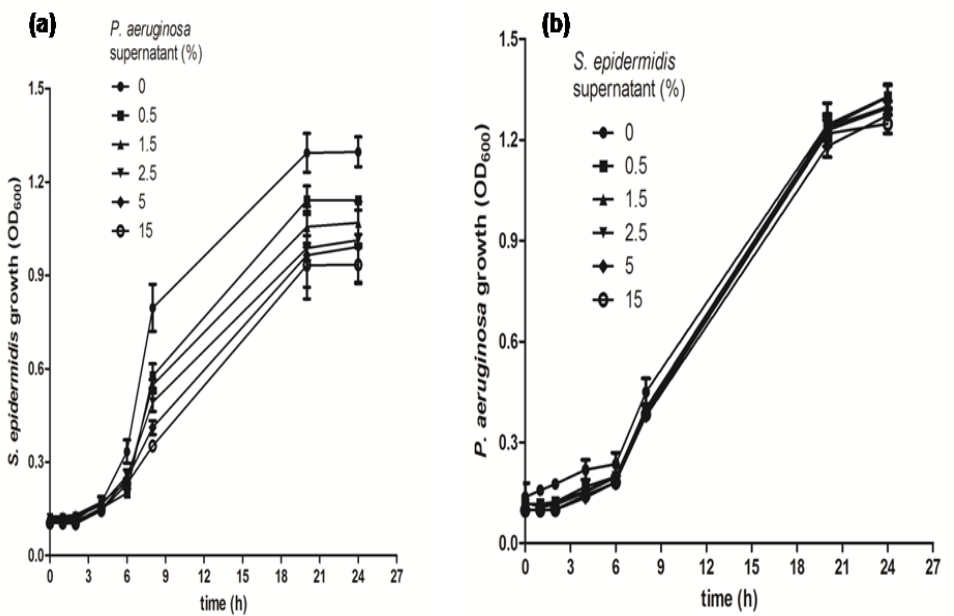

Figure 2(a) Effect of filter-sterilized P. aeruginosa supernatant on the growth of $S$. epidermidis and 2(b) indicates the effect of filtersterilized $S$. epidermidis supernatant on the growth of $P$. aeruginosa. Error bars indicate the \pm standard deviations of the measurements.

\subsection{Co-survival study in a biofilm system}

A mixed culture biofilm study was performed to understand the nature of interaction in biofilm mode. PAO1 presented higher biofilm than $S$. epidermidis $\left(\mathrm{OD}_{570}\right.$, mean $\pm \mathrm{SD}: 1.312 \pm 0.092$; PAO1 vs. $0.645 \pm 0.257$; SE1, $0.617 \pm 0.127$; SE2, $0.927 \pm 0.091 ;$ SE3, $1.038 \pm 0.159$ SE4, $1.054 \pm 0.197 \mathrm{SE} 5)$ as shown in Figure 3a. Moreover, the mixed culture biofilm level was significantly lower (net 1.4 fold) than PAO1 and similar to SE, which indicates that the presence of SE may affect the biofilm formation of PAO1. The increased biofilm biomass of mixed culture from M1-M5 indicates the enhanced persistence of SE in mixed culture.

\subsection{Microscopic analysis of mixed culture biofilm}

The microscopy was performed to analyze the interaction and variation in species-specific growth in mixed culture (Figure 3b). The single species culture biofilm demonstrated the abundant and dense colonization on glass coverslips under the light microscope and SEM. After $24 \mathrm{~h}$, PAO1, singlespecies biofilm was seen as adherent and immersed within EPS on the glass coverslips. SE control biofilm was seen in a cluster, either in pairs, tetrad or clumps, although EPS was scarcely visible. However, the reduction of PAO1 and SE biofilm cells was seen in M1 and M2 mixed culture compared to the control single culture counterpart. This signifies that in M1-M2 mixed culture, biofilm PAO1 and SE has a mutually suppressive effect on each other. Furthermore, as seen in M3M5 mixed culture, biofilm decreases the PAO1 cell number in contrast to its single control biofilm. While increasing EPS, uniform distribution, and increasing the accumulation of SE biofilm cells were seen as in M2-M5 mixed biofilm (Figure 3b). 

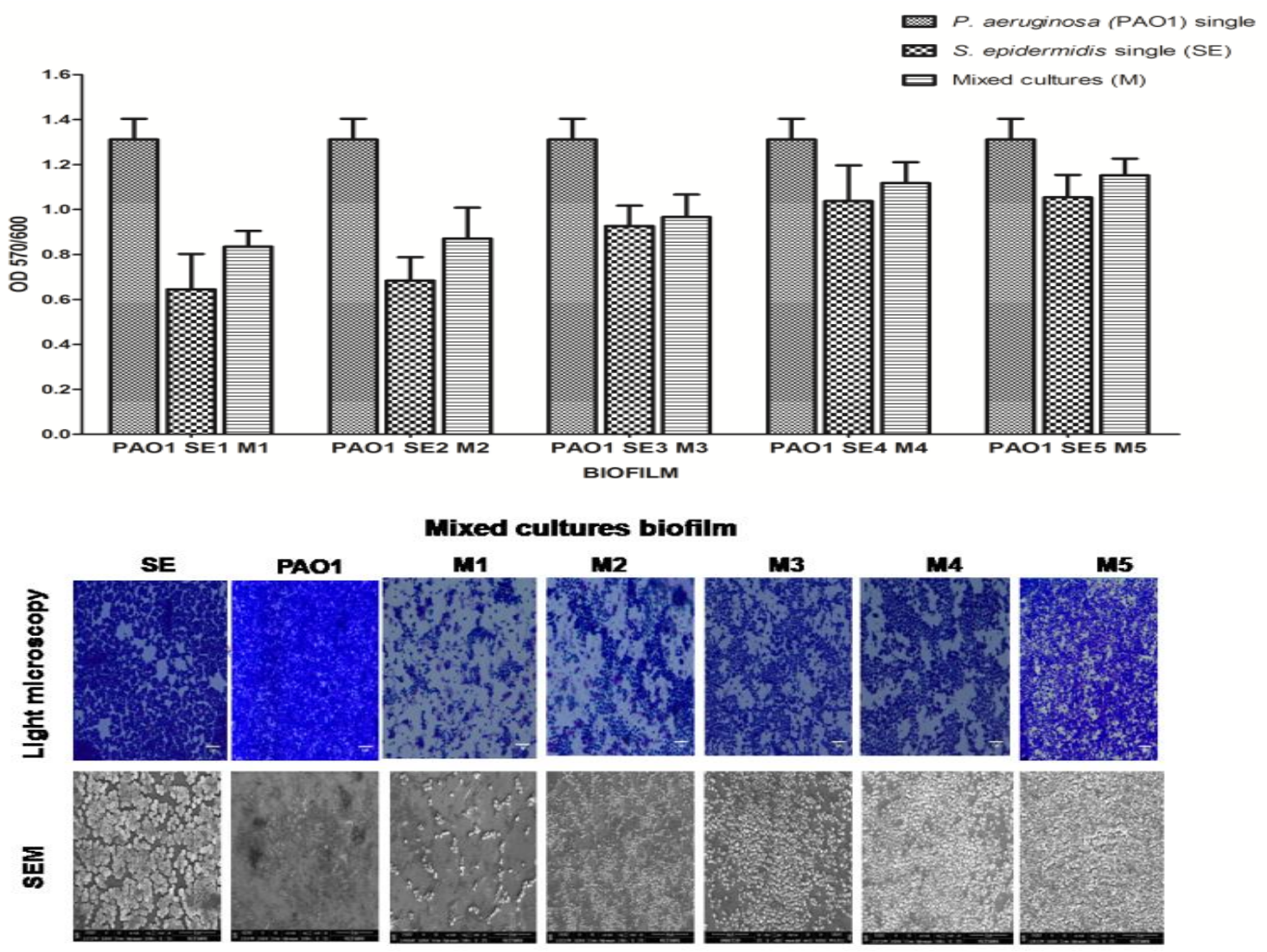

Figure 3(a) Quantification of single and mixed culture biofilm of $P$. aeruginosa and S. epidermidis by microtiter plate assay method. Where SE1, SE2, SE3, SE4, and SE5 indicate the different concentrations of $S$. epidermidis.M1-M5; mixed cultures biofilms of $P$. aeruginosa $\left(5.5 \times 10^{9} \mathrm{CFU} / \mathrm{ml}\right)$ with different concentrations of $S$. epidermidis $\left(5.5 \times 10^{9}, 6 \times 10^{9}, 7 \times 10^{9}, 8.5 \times 10^{9}\right.$, and $\left.1.5 \times 10^{10} \mathrm{CFU} / \mathrm{ml}\right)$.

Error bars indicate the \pm standard deviations of three measurements (b) Light microscopy (above lane) and scanning electron microscopy images (below lane) of mixed cultures biofilm of P. aeruginous and S. epidermidis. M1-M5; mixed cultures biofilms of $P$. aeruginosa with different concentrations of $S$. epidermidis.

(a)

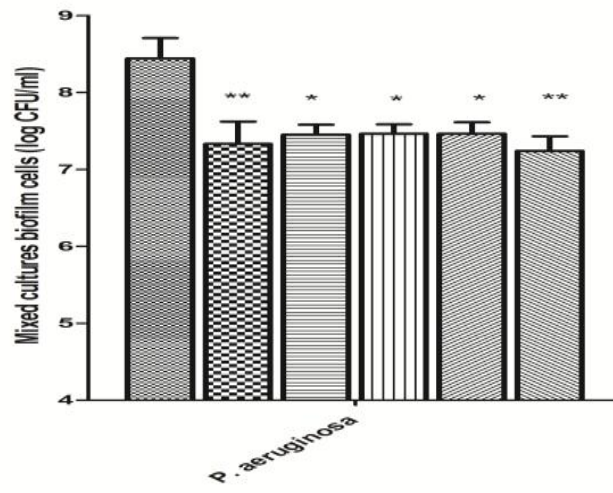

(b)

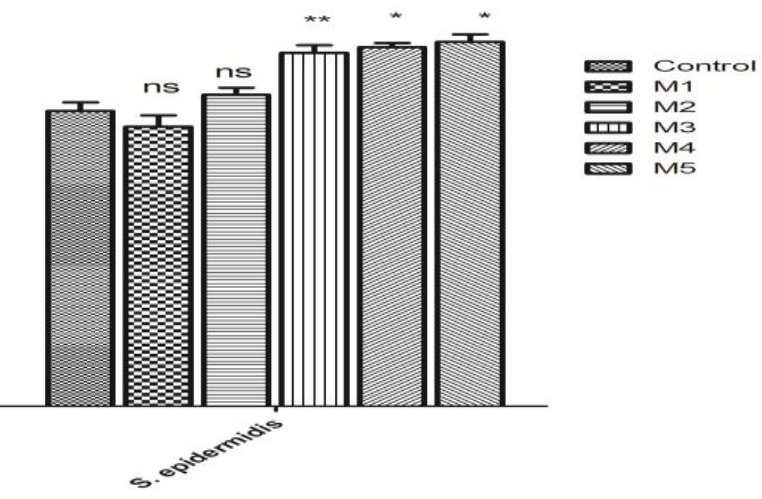

Figure 4 Biofilm cells of $S$. epidermidis and P. aeruginosa alone and in the mixed cultures (4a) Biofilm cells of $P$. aeruginosa in M1-

M5 biofilm compared with control (alone). (4b) Biofilm cells of $S$. epidermidis in the M1-M5 biofilm with control. $100 \mu 1$ standardized inoculums $\left(5.5 \times 10^{9} \mathrm{CFU} / \mathrm{ml}\right)$ are used in M1. Error bars indicate the standard deviations for three measurements.*, $\mathrm{P}<0.05$ compared with the control. **, $\mathrm{P}<0.001$ when compared with the control. 


\subsection{Analysis of cell viability of biofilm cells}

The biofilm viability was evaluated by the CFU count, as shown in Figure 4. When compared with the growth of single species biofilm, it was found that the viability of PAO1 and SE did not have a similar value in mixed biofilm. When compared to their CFU count of mixed culture biofilm, the viability (CFU) of biofilm formed by SE was higher than that of PAO1. When the viable count of SE was tested within mixed biofilms and compared with its single viability, its viability was observed to be significantly enhanced in M3-M5 mixed cultures biofilm and found similar in M1-M2 mixed biofilm (Figure 4b). On the other hand, the biofilm viability of PAO1 decreased in all sets of mixed biofilm (M1-M5), as shown in figure $4 \mathrm{a}$. Biofilm cell count of SE was less than $8 \mathrm{log}$ $(\mathrm{CFU} / \mathrm{ml})$, and PAO1 was noticed between 8 and $9 \log (\mathrm{CFU} / \mathrm{ml})$. The overall reduction in PAO1 biofilm cells was $88 \%$, and the promotion of SE biofilm was $75 \%$ to their control.

\subsection{Effect of bacterial supernatant on Biofilms}

Further, to understand the mechanism of reduction in PAO1 biofilm cells, whether it is a contact or non-contact basis, the effect of PAO1 supernatant on SE and vice versa were evaluated, and it was observed that biofilm of PAO1 was diminished in the presence of supernatant of SE (Figure 5a). Overall 1.1 fold reduction in PAO1 was observed as compared to control. Further, the reduction of PAO1 biofilm in the presence of cell-free supernatant of SE on the coverslip in a static condition was also evaluated (Figure $5 \mathrm{~b}$ ). After the fixation of biofilm, total cells involves in biofilm formation were stain with PI and visualized under fluorescent microscopy. The micrograph of PAO1 treated samples showed a scattered appearance in comparison to the $24 \mathrm{~h}$ PAO1 control biofilm. The biofilm cells attached to the surface were scattered, and cell packs were rarely observable because of poor cohesiveness and subsequent adherence. The biofilm integrity in terms of EPS production was additionally limited within the tested samples. On the other side, SE biofilm was enhanced in the presence of PAO1 supernatant in a concentration-dependent manner (Figure 6). The cell-free supernatant of PAO1 invigorates SE biofilm in a concentration-dependent way with a general, 2.6 fold increment in biofilm production as compared with the sample without PAO1 supernatant (Figure 6b). Data of microscopic visualization of SE biofilm with and without PAO1 cell-free supernatant revealed that SE biofilm (red) and EPS (green) increases as the supernatant concentration increases (Figure 6a).

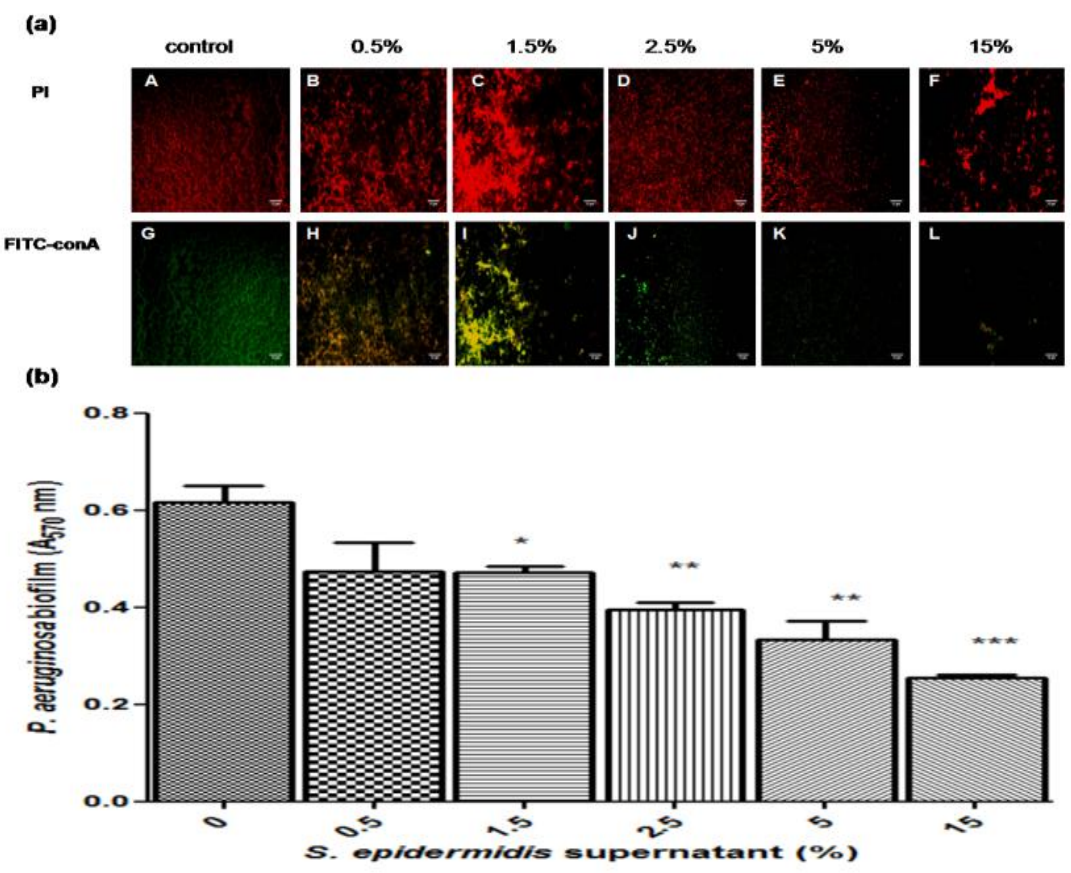

Figure 5 Inhibition of $P$. aeruginosa biofilm formation in the presence of cell-free culture supernatant of S. epidermidis. (5a); Microscopy images P. aeruginosa PAO1 biofilms in the presence of $0.5 \%, 1.5,2.5 \%, 5 \%$, and $15 \%$ (b, c, d, e, f, h, i,j, k, and l) and absence of $(\mathrm{a}, \mathrm{g})$ S. epidermidis 435 supernatant. Red fluorescent indicates the total cell involves biofilm, and green fluorescent, showing the EPS production in biofilm stained with FITC-ConA. (5b) Biofilm quantification of P. aeruginosa in the presence of $0.5 \%, 1.5,2.5 \%, 5 \%$, and $15 \%$ and without (zero \%) culture supernatant of S. epidermidis. Error bars indicate the standard deviations for three measurements. *, $\mathrm{P}<0.05$ compared with the control. **, $\mathrm{P}<0.001$ when compared with the control. ***, $\mathrm{P}<0.0001$ when compared with the control. 

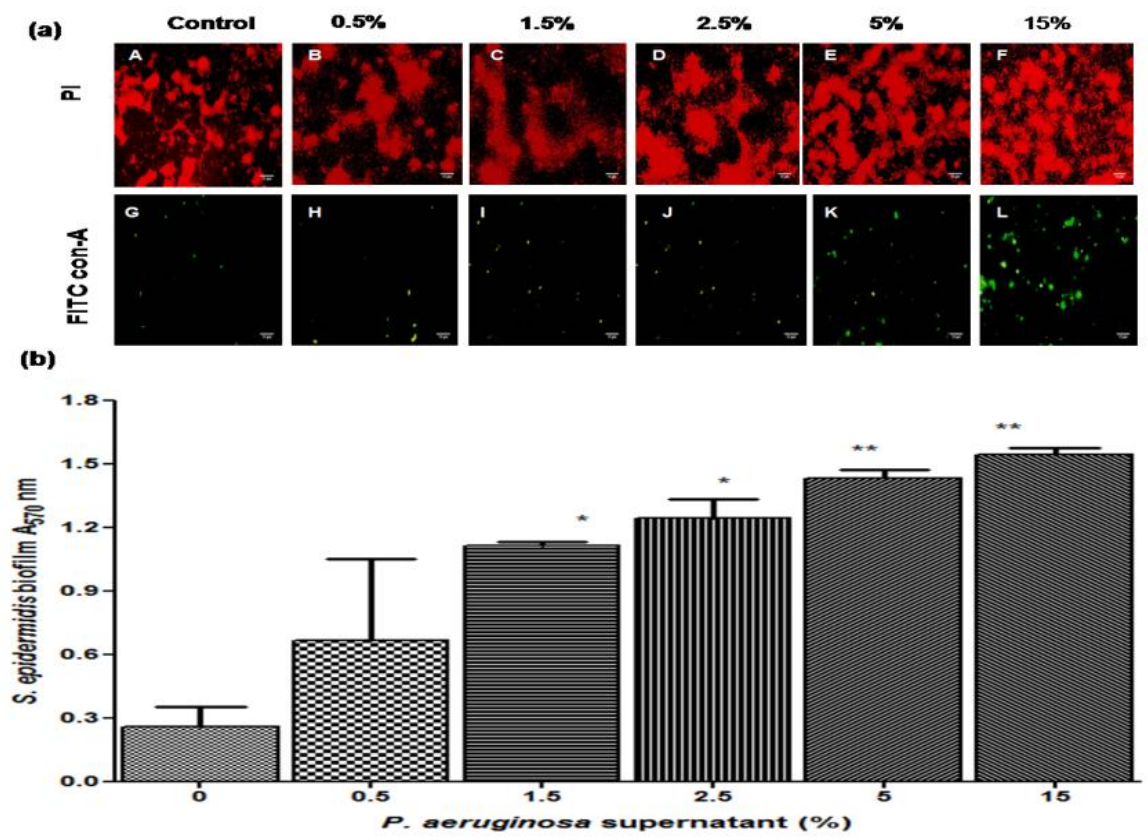

Figure 6 Biofilm formation of $S$. epidermidisin the presence of cell-free culture supernatant of $P$. aeruginosa. (6a) Microscopic images S. epidermidis biofilms formed with $0.5 \%, 1.5,2.5 \%, 5 \%$, and $15 \%$ (b, c, d, e, $\mathrm{f}, \mathrm{h}, \mathrm{I}, \mathrm{j}, \mathrm{k}$, and $\mathrm{l}$ ) and without (a, g) $P$. aeruginosa supernatant. Red fluorescence (a-f) indicates the total cell involves biofilm and green fluorescent, showing the EPS production in biofilm stained with FITC-ConA (g-1). (6b) S. epidermidis biofilm quantificationin the presence of $0.5 \%, 1.5,2.5 \%$, $5 \%$, and $15 \%$ and without (zero \%) culture supernatant of $P$. aeruginosa. Error bars indicate the standard deviations for three measurements. *, $\mathrm{P}<0.05$ compared with the control. **, $\mathrm{P}<0.001$ when compared with the control.

\section{Discussion and Conclusion}

The present study has evaluated the nature, and inter-bacterial interaction between PAO1 and SE435 in the planktonic and biofilm community, particularly emphasizes whose survivability enhanced to their planktonic counterparts. The agar plate assay, including both spot inoculation and equal half, streaked plates, zone of inhibition shows that the growth of SE was inhibited by PAO1. Additionally, the growth of SE was also inhibited by a culture supernatant of PAO1 (found net 0.7 , $0.8,1,1.1$, and 1.3 fold reduction). The antagonism behavior of bacterium could be due to the diffusible exoproducts released by PAO1and probably due to competition for nutrients and space in the same medium (Qin et al.,2009; Keown et al., 2020). Further analysis was carried out in the biofilm mode of growth. When both bacteria were cultivated alone, it was observed that, unlike SE biofilm, biofilm biomass of PAO1 (alone) was found net 2.8 fold higher, which may be due to increasing EPS production during biofilm formation. In mixed culture biofilm, it was observed that biofilm biomass of mixedspecies culture was similar to SE and lower than PAO1 individually, which suggests that perhaps SE persists in the mixed culture and affects the EPS production (Holt et al., 2017; Woods et al., 2019).
The study was further confirmed by microscopic analysis. Microscopic analysis was performed to analyze the interaction of PAO1 and SE in mixed-species biofilm formation, and it was observed that SE was more abundant than PAO1 in all sets of mixed cultures. The mixed cultures SEM images of SE and PAO1 biofilms showed that the enhanced EPS matrix of SE in comparison with PAO1. Light microscopy and SEM confirmed that the PAO1 showed scanty architecture in mixed-species biofilm instead of dense colonization as in single species. This confirmed that the dominance of SE over PAO1 in mixed-culture biofilms.

Moreover, the formerly mixed cultures (M1-M2) were also less dense and non-uniform than that of controls and revealed few layers of cells, profuse cellular debris, together with degrading and morphological alter PAO1 cells. The result is consistent with the findings of Bandara et al. (2010), who reported that both $P$. aeruginosa and Candida spp. mutually affect each other in dualspecies biofilm than single control biofilm. Further, the present investigation gave insights that complicated communications can occur between species inside the biofilm. Furthermore, the subsequent dynamic relies on various factors, for example, the microbial consortia, cell-cell communication, and the surface of attachment. SEM and light microscopy show that the total number of cells involves in biofilm formation, while only viable cells 
involve in biofilms were measured by CFU count using selective or differential agar media. Hence, with the SE cells concentration, a few scattered and scanty biofilm of PAO1 was confirmed by the CFU count. Besides, the current study also evaluated the SE biofilm viable cells in all sets of mixed cultures.

In this context, the viability of both species PAO1 and SE in M1M5 mixed biofilm was compared with single biofilm viable cells. It was observed that the viability of SE in M3-M5 was significantly higher than the control biofilm (single culture), as shown in figure 5a. While similar biofilm cells were observed in M1-M2 $(7.5 \log (\mathrm{CFU} / \mathrm{ml}) \pm 0.22)$. The possible explanation of enhanced survival in M3-M5 is that there may be an increased amount of $S$. epidermidis that allows the organism to out-compete the $P$. aeruginosa as compared to M1-M2. Although the population of $P$. aeruginosa PA14, either planktonic or biofilm, is not influenced by the presence of S. aureus (Filkins et al., 2015). At the same time, the outcomes of the current study demonstrate that the inhibitory activity of PAO1 against SE was only in planktonic form and the scanty existence of PAO1 as well as the promotion of SE biofilm in mixed culture biofilm. Similarly, Dehbashi et al., (2021), finding show that in co-culture biofilm state viability of $P$. aeruginosa bacteria decreased significantly with the presence of $S$. aureus.

Further, to understand the mechanism of reduction in PAO1 biofilm cells, whether it is contact or non-contact basis. The increase and decrease in the effect of SE and PAO1 supernatant, respectively, was investigated, and it was observed that SE supernatant reduced the PAO1 biofilm formation in mixed biofilm, and it was due to the secretion of extracellular products of SE. The proposed mechanism either interferes with the adhesion process of PAO1 or due to competition for surface attachment and nutrients within the same environment. On the other hand, the culture supernatant of PAO1 showed a positive impact on SE biofilm, as confirmed by the CV assay. Similarly, protease containing SE supernatant decreases the biofilm biomass of $S$. aureus (Vandecandelaere et al., 2014).

Similar results were obtained by fluorescent microscopy, and data confirmed that supernatant of SE interferes with the PAO1 biofilm formation and EPS production. On the other side, the SE-treated biofilm was enhanced in the presence of cell-free supernatant of PAO1 as compared to untreated biofilm. Moreover, a reduced biofilm of PAO1 was probably due to the presence of exogenous products such as proteases, nucleases, PNAG/PIA, and Phenolsoluble modulins (PSMs) functioning as surfactants in the supernatant of SE, which might interfere with adhering properties and pathogenicity of PAO1. Similarly, Kumari et al., (2019) findings also report the significant reduction of Rhizopus arrhizus spores and pre-existing hyphae with the exposure of phenol soluble modulins (PSMs) and S. epidermidis. Likely, Esp a serine protease delivered by S. epidermidis (Gram-positive commensal bacterium) prevents $S$. aureus biofilm formation, nasal colonization, and disperses preformed S. aureus biofilm (Iwase et al., 2010; Mitchell et al., 2010; Fugère et al., 2014).

Conversely, Lopes et al. (2011) demonstrated that metabolites present in $P$. aeruginosa supernatant stimulated the planktonic growth of $E$. coli and inhibited their biofilm formation. The overall data revealed that the survivability of $S$. epidermidis was higher in biofilm than in planktonic mixed and change the behavior of $S$. epidermidis from planktonic to biofilm form. This study supported that, in polymicrobial consortia, the pathogenicity and gene expression of pathogens can be altered by the presence of other bacterial species (Duan et al., 2003; Alves et al., 2018; Castro et al., 2019). The present study is important as it not only explores the nature of interspecies interaction but is also the first time we are reporting that biofilm inhibitory activity exhibited by the culture supernatant of SE435 is probably one of the clues to reduce or prevent the PAO1 associated biofilm infections.

In conclusion, this study analyses the interaction of $P$. aeruginosa with a mixed set of $S$. epidermidis in both planktonic and biofilm conditions. The findings show that the three mixed sets (M3, M4, and M5) can significantly reduced the $P$. aeruginosa biofilm growth. Additionally, in the case of competitive interaction where extracellular production of mix set M3, M4, and M5; increases the inhibitory activity of $S$. epidermidis against $P$. aeruginosa. Hence, the cell density controls the production of inhibitory molecules of $S$. epidermidis in mixed-species biofilm by cell density-dependent quorum sensing (cell to cell communication mechanism). Future research will involve identifying and developing potential inhibitors against resistant biofilm in vitro and in vivo.

\section{Declaration of Competing Interest}

The authors have declared that no conflict of interest exists.

\section{Acknowledgments}

The authors gratefully acknowledge the Ministry of Human Resource Development (MHRD) and TEQIP for financial support. The authors are also thankful to the IIT Kanpur for providing the SEM facility.

\section{References}

Alves PM, Al-Badi E, Withycombe C, Jones PM, Purdy KJ, et al. (2018) Interaction between Staphylococcus aureus and Pseudomonas aeruginosa is beneficial for colonisation and pathogenicity in a mixed biofilm. Pathogens and Disease 76(1):fty003. 
Baldan R, Cigana C, Testa F, Bianconi I, Simone MD, et al. (2014) Adaptation of Pseudomonas aeruginosa in cystic fibrosis airways influences virulence of Staphylococcus aureusin vitro and murine models of co-infection. PLOS ONE 9(3):e89614.

Bandara HM, Yau JYY, Watt RM, Jin LJ, Samaranayake LP (2010) Pseudomonas aeruginosa inhibits in-vitro Candida biofilm development. BMC Microbiology 1 0:125.

Cai P, Sun X, Wu Y, Gao C, Mortimer M, et al. (2019) Soil biofilms: microbial interactions, challenges, and advanced techniques for ex-situ characterization. Soil Ecology Letters 1(3):85-93.

Castro J, Machado D, Cerca N (2019) Unveiling the role of Gardnerellavaginalis in polymicrobial bacterial vaginosis biofilms: the impact of other vaginal pathogens living as neighbors. The ISME Journal 13(5):1306-1317.

Chabi R, Momtaz H (2019) Virulence factors and antibiotic resistance properties of the Staphylococcus epidermidis strains isolated from hospital infections in Ahvaz, Iran. Tropical Medicine and Health 47(1):1-9.

Dehbashi S, Alikhani MY, Tahmasebi H, Arabestani MR (2021) The inhibitory effects of Staphylococcus aureus on the antibiotic susceptibility and virulence factors of Pseudomonas aeruginosa: A549 cell line model. AMB Express 11(1):1-10.

Duan K, Dammel C, Stein J, Rabin H, Surette MG (2003) Modulation of Pseudomonas aeruginosa gene expression by host microflora through interspecies communication. Molecular Microbiology 50(5):1477-1491.

Filkins LM, Graber JA, Olson DG, Dolben EL, Lynd LR, et al. (2015) Coculture of Staphylococcus aureuswith Pseudomonas aeruginosadrives $S$ aureus towards fermentative metabolism and reduced viability in a cystic fibrosis model. Journal of bacteriology 197(14):2252-2264.

Fischer ER, Hansen BT, Nair V, Hoyt FH, Dorward DW (2012) Scanning electron microscopy. Current Protocols in Microbiology 2(2B):2- 2B.2.

Foster TJ (2020) Surface Proteins of Staphylococcus epidermidis. Frontiers in Microbiology 29;11:1829.

Fugère A, Séguin DL, Mitchell G, Déziel E, Dekimpe V, et al. (2014) Interspecific small molecule interactions between clinical isolates of Pseudomonas aeruginosaand Staphylococcus aureus from adult cystic fibrosis patients. PLOS ONE 9(1):e86705.
Hibbing ME, Fuqua C, Parsek MR, Peterson SB (2010) Bacterial competition: surviving and thriving in the microbial jungle. Nature Reviews Microbiology 8(1):15-25, 15-25.

Hoffman LR, Déziel E, D’Argenio DA, Lepine F, Emerson J, et al. (2006) Selection for Staphylococcus aureus small-colony variants due to growth in the presence of Pseudomonas aeruginosa. Proceedings of the National Academy of Sciences of the United States of America 103(52):19890-19895.

Holt JE, Houston A, Adams C, Edwards S, Kjellerup BV (2017) Role of extracellular polymeric substances in polymicrobial biofilm infections of Staphylococcus epidermidis and Candida albicans modelled in the nematode Caenorhabditis elegans. Pathogens and Disease 31:75(5).

Iwase T, Uehara Y, Shinji H, Tajima A, Seo H, et al. (2010) Staphylococcus epidermidis Esp inhibits Staphylococcus aureus biofilm formation and nasal colonization. Nature 465:346-349.

Joshi RV, Gunawan C, Mann R (2021) We Are One: Multispecies Metabolism of a Biofilm Consortium and Their Treatment Strategies. Frontiers in Microbiology 28;12:635432.

Kalia M, Singh D, Sharma D, Narvi SS, Agarwal V (2020) Senna alexandriana mill as a potential inhibitor for quorum sensingcontrolled virulence factors and biofilm formation in Pseudomonas aeruginosa PAO1.Pharmacognosy Magazine 16:797-802.

Keown K, Reid A, Moore JE, Taggart CC, Downey DG (2020) Coinfection with Pseudomonas aeruginosa and Aspergillus fumigates in cystic fibrosis. European respiratory review: an official Journal of the European Respiratory Society 29(158): 200011.

Kobayashi H, Oethinger M, Tuohy MJ, Procop GW, Bauer TW (2009) Improved detection of biofilm-formative bacteria by vortexing and sonication: A pilot study. Clinical Orthopaedics and Related Research 467(5):1360-1364.

Kumari A, Tewari R, Singh R (2019) Antagonistic interaction of Staphylococcus aureus and Staphylococcus epidermidis with Rhizopusarrhizus mediated by phenol soluble modulins and organic acids. ACS infectious diseases 5(11):1887-95.

Lopes SP, Machado I, Pereira MO (2011) Role of planktonic and sessile extracellular metabolic byproducts on Pseudomonas aeruginosa and Escherichia coli intra and interspecies relationships. Journal of Industrial Microbiology \& Biotechnology 38(1):133-140.

Mitchell G, Séguin DL, Asselin AE, Déziel E, Cantin AM, et al. (2010) Staphylococcus aureus sigma B-dependent emergence of 
small-colony variants and biofilm production following exposure to Pseudomonas aeruginosa 4-hydroxy-2-heptylquinoline- $\mathrm{N}$ oxide. BMC Microbiology 10:33.

Morales DK, Grahl N, Okegbe C, Dietrich LEP, Jacobs NJ, et al. (2013) Control of Candida albicans metabolism and biofilm formation by Pseudomonas aeruginosa phenazines. $\mathrm{mBio}$ 4(1):e00526-e00512.

Nithya C, Begum MF, Pandian SK (2010) Marine bacterial isolates inhibit biofilm formation and disrupt mature biofilms of Pseudomonas aeruginosa PAO1. Applied Microbiology and Biotechnology 88(1):341-58.

Otto M (2013) Coagulase-negative staphylococci as reservoirs of genes facilitating MRSA infection: staphylococcal commensal species such as Staphylococcus epidermidis are being recognized as important sources of genes promoting MRSA colonization and virulence. Bioessays 35(1):4-11.

Pang Z, Raudonis R, Glick BR, Lin TJ, Cheng Z (2019) Antibiotic resistance in Pseudomonas aeruginosa: Mechanisms and alternative therapeutic strategies. Biotechnology advances, 37(1):177-192.

Qin Z, Yang L, Qu D, Molin S, Tolker-Nielsen T (2009) Pseudomonas aeruginosa extracellular products inhibit staphylococcal growth and disrupt established biofilms produced by Staphylococcus epidermidis. Microbiology 155(7):2148-2156.

Rogers GB, Hoffman LR, Whiteley M, Daniels TWV, Carroll MP, et al. (2010) Revealing the dynamics of polymicrobial infections: implications for antibiotic therapy. Trends in Microbiology 18(8):357-364.

Singh PK, Yadav VK, Kalia M, Dohare S, Sharma D, et al. (2017) Pseudomonas aeruginosa auto inducer3-oxo- $\mathrm{C}_{12}$-HSL exerts bacteriostatic effect and inhibits Staphylococcus epidermidis biofilm. Microbial pathogenesis 110:612-619.

Thi MT, Wibowo D, Rehm BH (2020) Pseudomonas aeruginosa Biofilms. International Journal of Molecular Sciences 21(22):8671.

Vandecandelaere I, Depuydt P, Nelis HJ, Coenye T (2014) Protease production by Staphylococcus epidermidis and its effect on Staphylococcus aureus biofilms. Pathogens and Disease 70(3):321-331.

Woods PW, Haynes ZM, Mina EG, Marques CN (2019) Maintenance of $S$. aureus in co-culture with $P$. aeruginosa while growing as biofilms. Frontiers in microbiology 9:3291.

World Health Organization (2017) Prioritization of pathogens to guide discovery, research and development of new antibiotics for drug-resistant bacterial infections, including tuberculosis (No. WHO/EMP/IAU/2017.12). World Health Organization. 